\title{
Utilization of Physiologically Based Pharmacokinetic Modeling in Clinical Pharmacology and Therapeutics: an Overview
}

\author{
Courtney Perry ${ }^{1} \cdot$ Grace Davis $^{1} \cdot$ Todd M. Conner $^{1} \cdot$ Tao Zhang $^{1}$
}

Published online: 12 May 2020

(C) Springer Nature Switzerland AG 2020

\begin{abstract}
The purpose of this review was to assess the advancement of applications for physiologically based pharmacokinetic (PBPK) modeling in various therapeutic areas. We conducted a PubMed search, and 166 articles published between 2012 and 2018 on FDA-approved drug products were selected for further review. Qualifying publications were summarized according to therapeutic area, medication(s) studied, pharmacokinetic model type utilized, simulator program used, and the applications of that modeling. The results showed a 13-fold increase in the number of papers published from 2012 to 2018, with the largest proportion of articles dedicated to the areas of infectious diseases, oncology, and neurology, and application extensions including prediction of drug-drug interactions due to metabolism and/or transporter-mediated effects and understanding drug kinetics in special populations. In addition, we profiled several high-impact studies whose results were used to guide package insert information and formulate dose recommendations. These results show that while utilization of PBPK modeling has drastically increased over the past several years, regulatory support, lack of easy-to-use systems for clinicians, and challenges with model validation remain major challenges for the widespread adoption of this practice in institutional and ambulatory settings. However, PBPK modeling will continue to be a useful tool in the future to assess therapeutic drug monitoring and the growing field of personalized medicine.
\end{abstract}

Keywords Physiologically based pharmacokinetic modeling · PBPK modeling applications $\cdot$ Modeling and simulation · In vitro in vivo extrapolation $\cdot$ Personalized medicine

\section{Introduction}

Our healthcare system is transitioning towards a patientcentered healthcare model, in which therapies are selected on the basis of a patient's needs and preferences and optimized according to patient-specific factors including age, body mass, allergies, and disease states. In this model, healthcare providers, including physicians, nurses, and pharmacists, work

Courtney Perry and Grace Davis contributed equally to this work.

This article is part of the Topical Collection on Precision Medicine and Pharmacogenomics

Electronic supplementary material The online version of this article (https://doi.org/10.1007/s40495-020-00212-x) contains supplementary material, which is available to authorized users.

Tao Zhang

zhangt@husson.edu

1 School of Pharmacy, Husson University, Bangor, ME 04401, USA together to provide care, ensure patient safety, reduce medication errors, and improve clinical outcomes. Physicians must individualize therapies to a patient by examining factors such as age, disease states, allergies, and concurrent medications. Pharmacists also play a crucial role in the identification, resolution, and prevention of medication-related problems such as medication overuse and underutilization, as well as the prevention and detection of adverse drug reactions and adverse effects.

An adverse drug reaction (ADR) is defined as "any response to a drug that is noxious and unintended, and that occurs at doses used for prophylaxis, diagnosis, or therapy, excluding failure to accomplish the intended purpose" [1]. ADRs are distinguishable from adverse effects, which are "expected, well-known reactions resulting in little or no change in patient management". Medication adverse effects and adverse drug reactions are studied during the drug approval process and data on their frequency and severity are included in the product's package insert, while those detected after a product has reached the market can be reported to the FDA 
through the MedWatch program. This allows providers to effectively communicate known reactions to patients and provide appropriate monitoring. An additional consideration for providers is the medication adverse effects which are provoked by a patient's comorbidities (e.g., renal and hepatic impairment) and interactions with other medications. Drugdrug interactions (DDIs) lead to changes in the pharmacokinetics of treatments, resulting in unintended ADRs, an increase in adverse effects, or reduced efficacy. This is especially important with narrow therapeutic index drugs such as warfarin, lithium, and digoxin, because even minimal changes in drug concentration or metabolism can lead to the drug not being effective or can cause toxic and potentially irreversible adverse effects. Conversely, exploitation of drug interactions may be beneficial in some treatment regimens; for instance, utilization of cobicistat (an enzyme inhibitor) to increase the exposure of atazanavir or darunavir in the treatment of human immunodeficiency virus (HIV). Although the pharmacist acts as a late gatekeeper for drug efficacy and safety, utilization of pharmacokinetic/pharmacodynamic (PK/PD) modeling tools may be helpful to facilitate the decision-making process in all levels of the patient-centered healthcare model.

Physiologically based pharmacokinetic and pharmacodynamic (PBPK/PD) modeling has become more prevalent to aid with the prevention of adverse drug events, drug-drug interactions, and drug-disease interactions. PBPK modeling is a mechanistic mathematical modeling technique that integrates drug (substance) and system (physiology) information that is used to predict pharmacokinetic characteristics and disposition for drugs [2,3]. Differential equations are implemented in a number of commercial software packages (e.g., Simcyp ${ }^{\circledR}$ Population-Based Simulator, GastroPlus ${ }^{\circledR}$, and PK-SIM ${ }^{\circledR}$ ) [4]. PBPK models provide many advantages over static models, which typically use one or two in vitro parameters to predict specific human PK parameters, overall drug exposures, or magnitude of drug-drug interactions (DDI) without considering time-varying changes [5]. In contrast, PBPK models are able to assess complex clinical scenarios, such as potential DDIs due to time-dependent metabolism or transporter changes, pharmacokinetics in special populations with notable physiological differences (e.g., pediatrics, geriatrics, and pregnancy), bioequivalence, food effects, and pharmacogenomics [6]. By incorporating drug-specific and system-specific parameters from different sources, these models are able to estimate PK parameters, further our understanding of a compound's properties, and predict plasma and tissue concentration-time profiles.

PBPK modeling can be an important tool for healthcare professionals to create individualized drug therapy regimens by assisting them in choosing the optimal dose, frequency, and route of administration for their patients based on population pharmacokinetic information. There are many individualized parameters that can alter a drug's absorption, distribution, metabolism, and elimination (ADME) characteristics, including age, comorbidities, renal and hepatic status, genetic polymorphisms, and more. PBPK modeling is a predictive tool that can be used to influence drug choice, selection, and routes of administration in different ethnic populations as well as populations of various ages and with various disease stages. Some applications of PBPK models include examination of potential drug-drug interactions, pharmacokinetics within special physiological populations (e.g., pediatrics, pregnancy), and other pharmacokinetic differences based on internal (genetic polymorphisms) and external (food effect) characteristics. Through the use of modeling, clinical pharmacists can play a role in the management of complex drug regimens and may be able to avoid drugs with potential adverse effects in susceptible patients.

Simulation of drug disposition and PK prediction has evolved as an important instrument in drug development, regulatory review, and clinical study design [7]. When submitting a New Drug Application (NDA), researchers can use PBPK predictions to fill in unknown clinical gaps during the evaluation of a breakthrough therapy drug. The U.S. Food and Drug Administration (FDA) recently announced the availability of a guidance document concerning format and content of PBPK data in regulatory submissions [8]. The FDA guidelines suggest the use of PBPK modeling in DDI assessment and hepatic impairment studies. In addition, the European Medicines Agency has released their own guidance for the utilization of PBPK modeling in research, which mandates a detailed report of the model, including drug-specific parameters, an overview of the model building process, sensitivity analyses, and a discussion on the regulatory impact of the modeling be submitted along with all results and other relevant data [9]. PBPK modeling allows the pharmaceutical industry, regulators, and academic researchers to overcome several challenges in creating a new drug and allows them to create an efficient and economical way to ensure the safety and effectiveness of the medication.

The objective of our work was to examine recently published PBPK modeling studies in order to profile their applications in various therapeutic areas and to assess their relevance to clinical practice. We also aim to highlight some of the high-impact applications of PBPK modeling and discuss the regulatory factors which support ongoing research in this field.

\section{Literature Search Strategy}

We conducted a search through PubMed using the search terms "Physiologically based pharmacokinetic modeling" and "Physiologically based pharmacokinetic-dynamic modeling" within the title or abstract of an article. Manuscripts were selected for assessment if they were written in English 
between 2012 and 2018 and presented PBPK models established/validated with human (clinical) PK data on FDA-approved products. Articles for medications that were still in clinical testing were not included. However, the quantity of publications cited may not adequately represent all the PBPK/PD models because of the restrictions used.

Qualifying publications were categorized into the following therapeutic categories: cardiovascular, endocrine, gastrointestinal, hepatic, immunosuppressants, infectious disease, neurologic, oncology, renal, respiratory, and supplements. Furthermore, each medication was classified by drug class, PK model type utilized (e.g., PBPK, PBPK/PD), and the specific simulator program employed. Specific simulator programs included PK-Sim, QSP, MATLAB, GastroPlus, Monolix 3.2, and Non-MEM. The model applications for drugs in each article were also categorized by drug-drug interactions due to either metabolism, transporter effects, or combinations of both. Other applications listed here include special populations (e.g., renal impairment, pregnancy, pediatrics, race and ethnicity) and pharmacokinetic prediction (e.g., pharmacogenetics, bioequivalence, tissue distribution, food effects).

\section{Results}

The idea of simulating PK by using multicompartment models with physiological factors was introduced by Teorell in 1937 , but PBPK was not widely used until recently [10, 11, 12]. One key factor is the increasing availability of in silico and in vitro systems, which act as surrogates for in vivo ADME processes and the advancement of in vitro to in vivo correlation of these data.

Our literature search resulted in $166 \mathrm{PBPK}$ or PBPK/PD manuscripts published since 2012 which met the criteria, excluding publications of medications in development. A summary of each article summarized by therapeutic area, medication, application, software, model type, and year are shown in Tables 1 through 5 in Online Resource 1. The majority of PBPK models have been developed for infectious disease, accounting for $24 \%$ of all articles, followed by oncology at $22 \%$, neurology at $18 \%$, cardiovascular at $17 \%$, and endocrine with $5 \%$. Therapeutic areas representing altogether $\leq 14 \%$ were renal, hepatic, respiratory, gastrointestinal, immunosuppression, supplements, and miscellaneous (Fig. 1). In addition, the number of articles published in each year increased from 4 in 2012 to 53 in 2018, representing a 13-fold increase (Fig. 2).

Modeling related to infectious disease accounted for the largest portion of modeling literature, comprising 40 of the 166 eligible articles. The number of articles increased from 1 article published in 2012 to 16 articles published in 2018 , representing a 16-fold increase. A likely reason for the increase in articles is due to increased concern about antibiotic resistance and the involvement of anti-infectives in clinically significant drug-drug interactions. According to the World Health Organization, antibiotic resistance is one of the biggest threats to global health, food security, and development today. Resistance to antibiotics has been attributed with the inappropriate use and overuse of antibiotics in humans and animals, and it has resulted in longer hospital stays, higher medical costs, and increased mortality. Drug-drug interactions are of particular clinical importance with antimicrobials, especially in patients who are receiving therapy for HIV. Roberts et al. examined the DDI between antiretrovirals and antituberculotic drugs in patients with HIV coinfected with Mycobacterium tuberculosis [13]. Resistance to antituberculosis drugs can occur when prescribers prescribe the wrong treatment or the wrong dose for an inappropriate length of time. The treatment of tuberculosis is especially critical in patients with HIV because patients are immunocompromised, which increases the mortality rate of infections. One of the common drugs used for tuberculosis in patients with HIV is rifampin, an antibiotic that acts as a strong inducer for many cytochrome P450 enzymes, which in turn can lower the expected concentrations of drugs which are hepatically metabolized. PBPK modeling to assess the DDI risk of antituberculosis drugs like rifampin when co-administered with other medications is incredibly important as resistance may develop with low drug concentrations.

Papers related to oncologic medications accounted for 36 of the 166 papers. The number of articles in this area increased from 2 published in 2012 to 14 published in 2018, representing a 7-fold increase. According to the American Cancer Society, it is estimated that there will be $1,735,350$ new cancer cases diagnosed and 609,640 cancer deaths in the USA in 2018. Per the National Cancer Institute, cancer is the second largest cause of mortality within the USA, following behind cardiovascular diseases, and approximately $38.4 \%$ of men and women are diagnosed with cancer during their lifetimes. New research is constantly being done to create better treatments for a variety of cancer types, and PBPK models have the potential to play a major role in the approval of new drugs. Many oncologic medications have severe adverse effects, so it is unethical to treat healthy subjects in live clinical trials. In this application setting, PBPK modeling has been used to determine adverse effects, dosing strategies, and the potential for drug-drug interactions. In addition, it can be used to determine optimal dosing strategies based on comorbidities and other patient-specific factors. Several notable applications of PBPK modeling profiled here include determination of the dosing of etoposide in adults and children and systemic drug exposure of busulfan in children [7, 14].

According to the CDC, cardiovascular diseases are the number one cause of mortality within the USA. Within the USA, 77.9 million (1 out of every 3 ) adults have hypertension. 


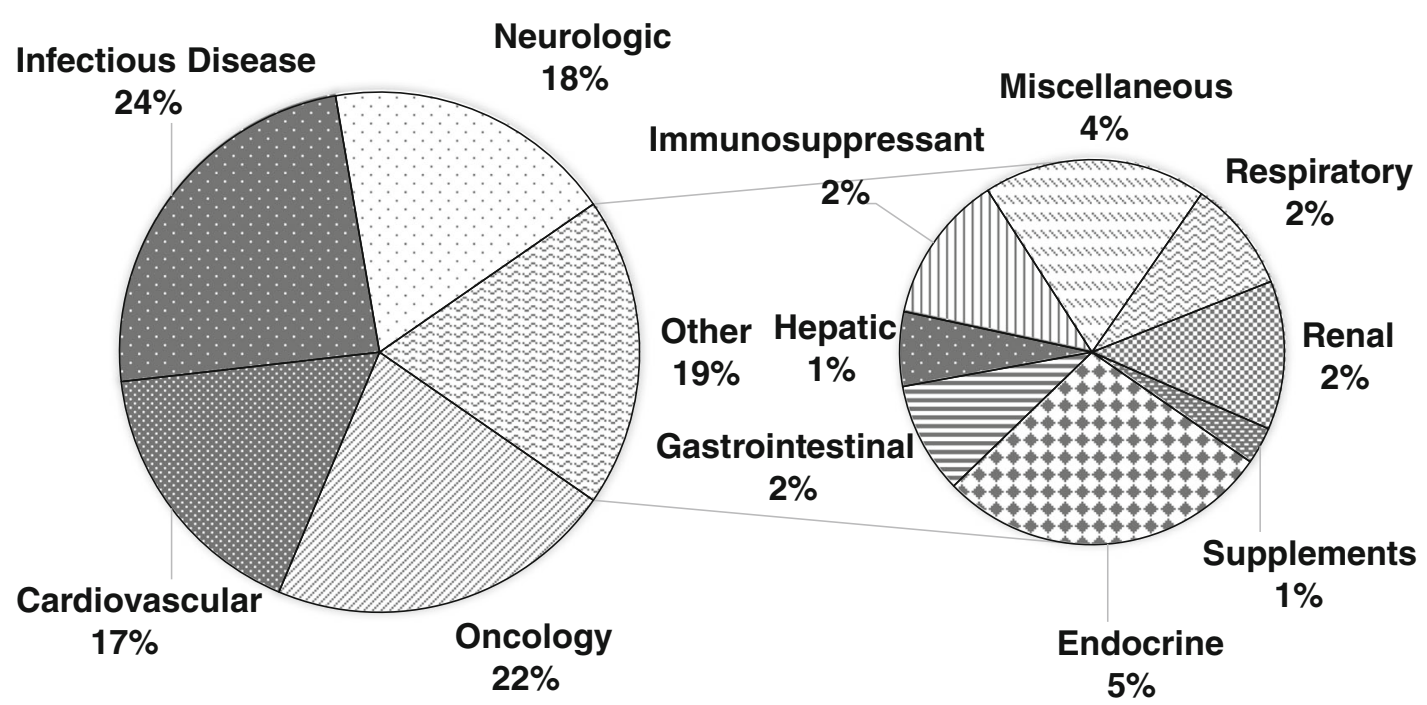

Fig. 1 Percentages of articles in each therapeutic area published between 2012 and 2018 utilizing PBPK modeling on FDA-approved products

This number has recently increased with the release of updated guidelines by the ACC/AHA, which have placed more stringent definitions on optimal blood pressure in adults. As such, it was anticipated that this therapeutic area would have one of the highest proportions of articles. Applications involving cardiovascular drugs represented 28 out of 166 articles published from 2012 to 2018 . The number of articles focused on cardiovascular medications increased from 1 article in 2012 to 10 articles in 2018, a 10-fold increase.
Neurologic drugs also comprised a significant portion of the medications studied using PBPK modeling and accounted for 30 of the 166 articles. This represents an increase from 1 article published in 2012 to 10 articles published in 2018, a 10 -fold increase. Drugs which act on the central nervous system are of great interest in PBPK modeling, as their activity depends on adequate levels of drug within the target tissue (e.g., brain). Because of this, clinicians often rely on surrogate markers of plasma concentration in order to determine a dose-

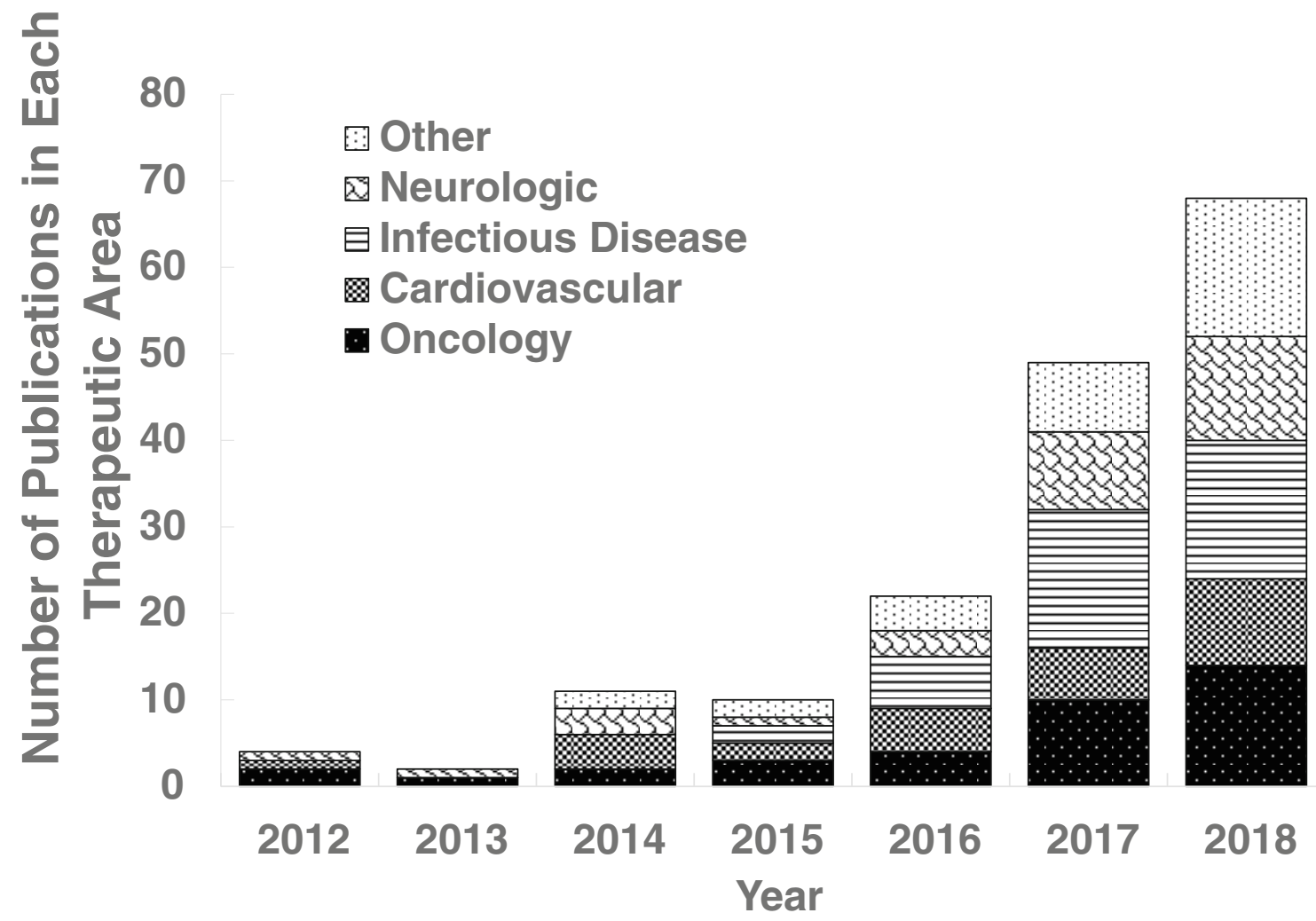

Fig. 2 Total number of articles in each therapeutic area published between 2012 and 2018 utilizing PBPK modeling on FDA-approved products 
related exposure-response relationship [15]. The blood-brain barrier consists of tight junctions between capillary endothelial cells, which restrict diffusion into the central nervous system, which even once entered can be removed by efflux pumps (e.g., P-glycoprotein or MDR1, and breast cancerresistant protein) $[16,17]$. The ability of a drug to penetrate this barrier is essential in the treatment of central nervous system (CNS) diseases including epilepsy, psychiatric disorders, and neurodegenerative disorders. In addition, drugs indicated for non-neurologic diseases, such as efavirenz (an antiviral) may have enhanced permeability into the CNS and produce serious adverse effects such as neurocognitive impairment. This creates marked importance on the utilization of quantitative approaches like PBPK modeling in order to predict drug disposition in both the plasma and target tissue.

Figures 3 and 4 show the majority of articles about model applications pertained to drug-drug interaction (DDI) prediction (34\%). This was further classified into metabolism $(23 \%)$, transporter (7\%), and combination applications (4\%). Assessment of DDI through modeling allows health care providers to examine the interaction potential of medications and interpret their clinical significance without causing patient harm. Other notable applications included pharmacokinetic predictions $(26 \%)$ and simulations in special populations $(30 \%)$. Special populations were broken into subgroups including renal impairment, pregnancy, pediatrics, and race/ethnicity. Other model applications representing altogether $9 \%$ of articles included cerebrospinal fluid distributions, impact of genetic polymorphisms, bioequivalence, and pharmacokinetic variability. PBPK was the most common model type seen in the literature search (93\% of all articles); a drastically fewer percentage of models included a pharmacodynamic component as well (7\%). Several articles were found with application extensions that included dose recommendations and patient package insert changes, simulation of challenging clinical scenarios (e.g., impact of a missed dose), and providing guidance for clinical trials which are currently underway,

PBPK modeling offers distinct advantages over other types of mechanistically static models (i.e., utilizing one or two in vitro parameters to predict discrete human pharmacokinetics), which rely on steady-state presumptions. PBPK is dynamic in the ability to simultaneously model multiple drug disposition processes [18-20]. We have highlighted, within applications for each disease state, several mechanisms specific to PBPK modeling that enhance understanding of drug product mechanisms. These include (1) prediction of drug concentrations in specific target tissues, (2) generation of virtual clinical studies to demonstrate variation in outcomes in regard to genetic polymorphisms, (3) drug-disease modeling for high-acuity clinical situations, (4) prospective prediction of drug pharmacokinetics and dosing using in vitro to in vivo extrapolations (IVIVE), and drug absorption, especially for advanced drug delivery systems (i.e., controlled- or extended-release).

\section{Application for Infectious Disease}

A major area of focus for PBPK modeling in infectious diseases is the prediction of pharmacokinetics in special populations; specifically, pediatric and geriatric patients. Schlender et al. used a PBPK model to evaluate the predictive performance of ciprofloxacin in pediatric and geriatric patients outside of the tested adult age range [21]. Claassen simulated amikacin and paracetamol drug levels in preterm neonates, a patient population which requires extreme care with medication dosing [22]. The researchers concluded that PBPK simulation in preterm neonates has the potential to be a useful tool in the future to support dosing decisions. Hornik et al. used modeling to demonstrate the feasibility of using pediatric data to develop pediatric PBPK models, thus extending the reach of this powerful modeling tool [23].

Another important area of interest in infectious disease is HIV. Patients with HIV are often prescribed complex, multidrug regimens which present with a multitude of drug-drug interactions. Without clinical data, DDIs are often predicted based on preclinical data and knowledge of the disposition of individual drugs. Predicting drug interactions can be extremely difficult because some antiretroviral drugs induce and inhibit multiple cytochrome P450 isoenzymes simultaneously. This can present a significant challenge for clinicians, who are tasked with monitoring not only the patient's antiviral regimen but also providing appropriate prophylaxis against opportunistic infections. Roberts et al. created a model to examine the treatment of Mycobacterium tuberculosis in patients with HIV-infected patients [13]. The aim of this study was to quantify the effect of cobicistat or ritonavir in modulating DDIs between darunavir and rifampin in a human hepatocyte-based in vitro model. Another study sought to predict the magnitude of the DDI between efavirenz, an inducer of CYP3A4 and inhibitors of CYP2C8, and dual CYP3A4/CYP2C8 substrates (repaglinide, montelukast, pioglitazone, paclitaxel) using PBPK modeling [24]. Another study used a PBPK model of the interaction between levonorgestrel and efavirenz in order to develop a dose-adjustment strategy for overcoming this interaction [25].

PBPK modeling has the distinct advantage of predicting drug concentrations in target tissues to help guide development of dosing regimens for specific diseases. Regarding infectious diseases, a current example of this can be seen with Yao et al. and their development of PBPK models for chloroquine and hydroxychloroquine to evaluate oral dosing regimens for SARS-CoV-2 infection [26]. A permeability rate limiting model and high lung-to-plasma partition coefficient was used to imitate drug accumulation in lung tissue, the 
45

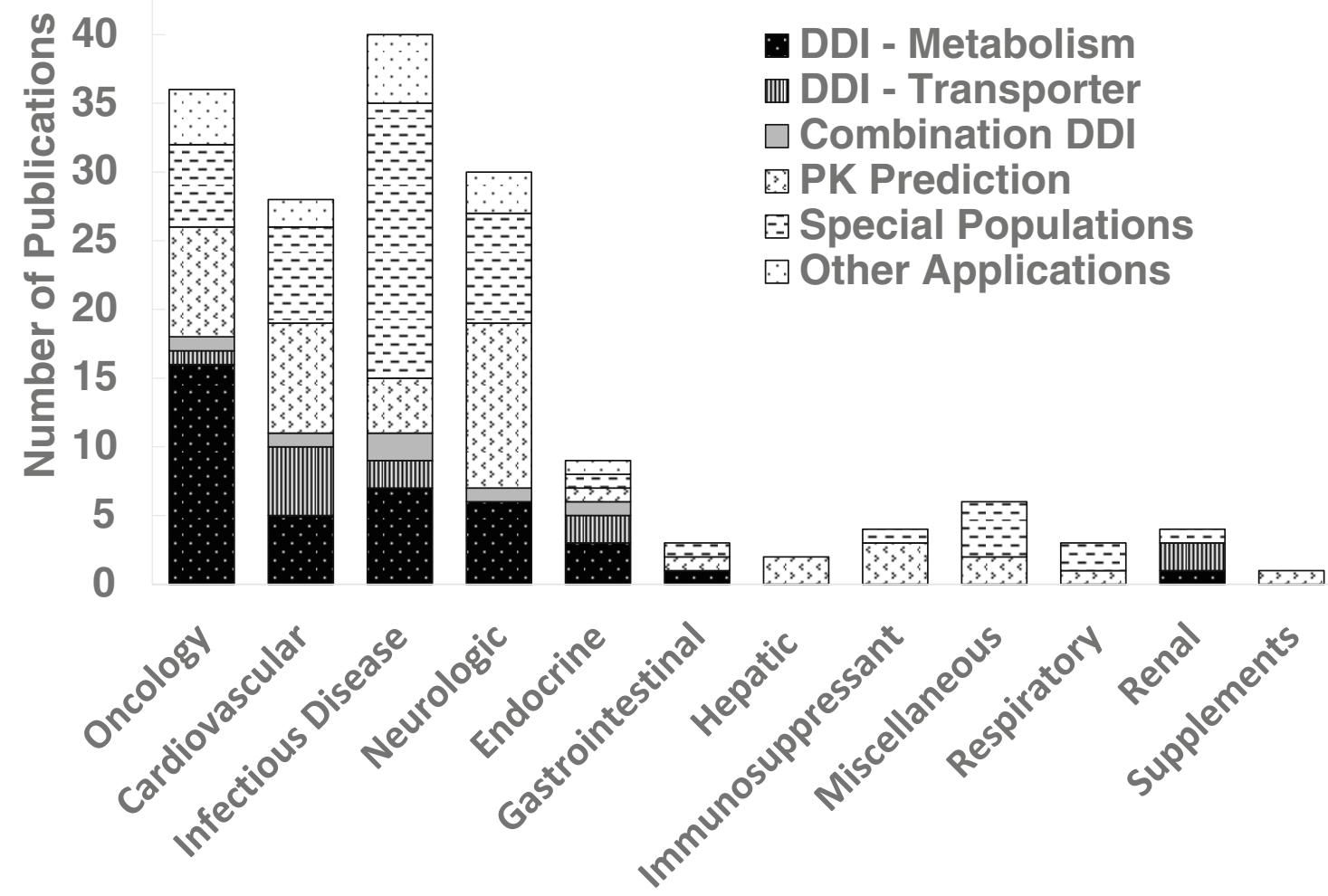

Fig. 3 Applications of PBPK modeling in various therapeutic areas

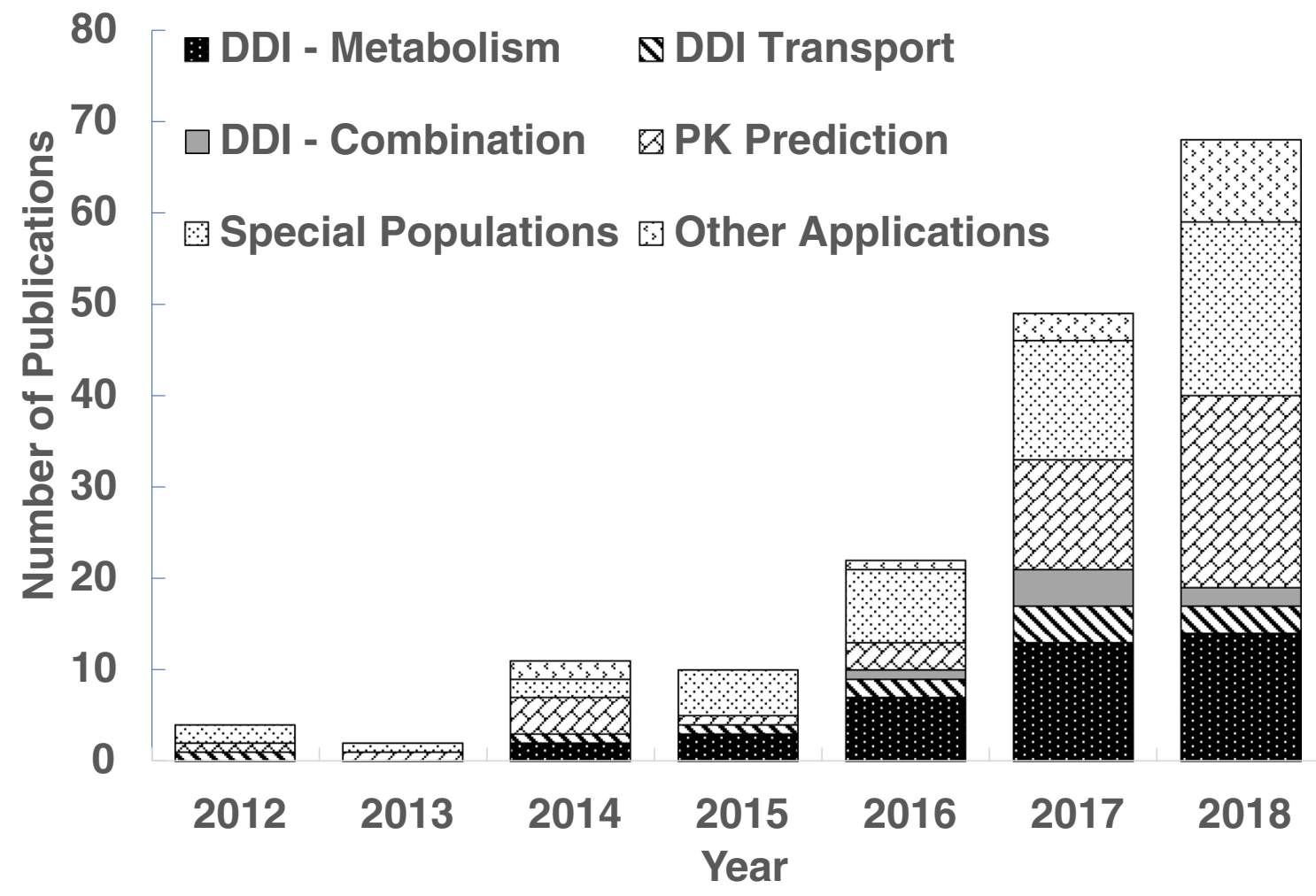

Fig. 4 Trends in applications of PBPK modeling from 2012 to 2018 
investigated site of action in COVID-19 to reduce potential for cytokine storm by IL-6 and IL-10 which results in multi-organ failure. Several clinical trials are investigating both drugs for use in SARS-CoV-2 infection and as such, the PBPK model provides the groundwork for an optimized dosing regimen for clinical trials due to evaluation of the unique pharmacokinetics (i.e., high accumulation in cells and long elimination halflife) to maximize lung tissue concentrations while minimizing the systemic adverse effect profile. The assessment of target tissue concentrations is an advantage specific to PBPK models as they are equipped to assess anatomic (e.g., volumes and tissue compartment connectivity) and physiologic (e.g., flows and clearance) characteristics of molecular entities while also considering the physicochemical properties of the drug [27].

\section{Application for Oncologic Medications}

PBPK models play a major role in the investigation and approval of oncologic medications. Because agents used to treat cancer target rapidly dividing cells and thus have an unfavorable adverse effect profile, it may be unethical or impractical to treat healthy subjects in clinical trials, which increases the utility of PBPK modeling for this application. The therapeutic classes of oncologic medications investigated included topoisomerase I/II inhibitors, histone deacetylase inhibitors, alkylating antineoplastic agents, monoclonal antibodies, selective estrogen receptor modulators (SERMs), and hedgehog pathway inhibitors. Understanding drug-drug interactions and special populations were the major applications seen with oncologic medications, reflecting the scenarios that clinicians most often encounter in practice. In addition to antineoplastic agents themselves, regimens frequently consist of "comfort medications," including anti-nausea medications, appetite stimulants, etc., which are used to make the patient's chemotherapy regimen more tolerable but can also impact drug metabolism and result in adverse effects. Lastly, cancer patients may have other comorbid conditions or organ damage which can be due to the type of cancer or chronic consequences of the other medications they are taking.

Several studies involving oncologic medications focused on interactions mediated through the cytochrome P450 (CYP450) enzymatic network. Narayanan et al. studied CYP3A4 induction by enzalutamide via Simcyp, which is a therapy approved for the treatment of metastatic prostate cancer and is often dosed as a combination in clinical practice [4]. Einolf et al. examined the interaction between ketoconazole and rifampin with the use of sonidegib, a chemotherapeutic agent used in the treatment of basal cell carcinoma which is also a CYP3A4 substrate. [28]. Studies conducted by Budha et al. and Yamazaki on cobimetinib and crizotinib respectively explored the impact of drug-drug interactions involving CYP3A4 substrates and evaluated the results using Simcyp
[29, 30]. In addition, Simcyp was utilized to examine the potential for drug-drug interactions involving renal organic anion transporters (OATs) in patients using pemetrexed [31].

Virtual clinical studies (VCS) are a considerable asset of PBPK models as they enhance understanding of the variation in clinical outcomes for a drug within a specific population. Parameter sets may be generated randomly to reflect the variation in pharmacokinetic and physiologic properties within a specific population, mainly by Monte Carlo methods. In this method, each "virtual subject" is assigned a unique set of these properties while maintaining the distribution of genetic polymorphism, ethnic differences, and intra- and inter-individual variability for that population. An example of this is best depicted by Toshimoto et al. and their assessment of irinotecan administered intravenously using VCS to identify factors related to drug-induced adverse effects (e.g., neutropenia and diarrhea) [32]. Correlation between drug exposure and adverse effects of neutropenia and diarrhea was determined through simulated AUC values above a set AUC threshold in plasma and enterocytes, respectively, which were derived from in vivo studies. In this work, 1,000,000 virtual subjects were generated and AUC values of irinotecan and its metabolite (SN-38) were simulated in the context of 6 genetic polymorphisms, including UGT1A1*28 and SLCO1B1 c.521 T > C. The probability of adverse effects in the virtual population were compared against reported values and found to be similar, which emphasized the importance of genetic polymorphism on irinotecan-induced adverse effects.

Similarly, a VCS with 24,000 patients by Dickschen et al. emphasized the impact of drug holidays of different lengths on steady-state kinetics and plasma levels of tamoxifen and its active metabolite, endoxifen, in patients with different CYP2D6 genotypes (i.e., extensive, intermediate, and poor metabolizers) [33]. The VCS explores the impact of patient adherence, which is difficult and nearly impossible to assess prospectively in vivo as patients tend to declare their compliance greater than what is reality. The study results support the use of a fixed-dose combination of tamoxifen and endoxifen in CYP2D6 extensive- and intermediate-metabolizers in combination with therapeutic drug monitoring as drug holidays were found to tremendously decrease plasma levels despite the long half-life of tamoxifen. Both aforementioned studies demonstrate the marked power and wealth of information that VCS from PBPK modeling can have when performed retrospectively and prospectively in differing populations and clinically important situations.

There was a large percent of articles that focused on modeling on special populations. Walsh et al. used PBPK to predict exposure of actinomycin D in younger patients [6]. PK-SIM was used to study drug exposure in children and adults in two key studies. Diestelhorst et al. used PK-SIM software to determine the first dose of busulfan in children via PBPK model parameterization [7], while Kersting et al. used PK-Sim 
software to evaluate the ability of PBPK modeling to predict the systemic drug exposure of high- and low-dose etoposide in children from a model developed with adult data [14]. Two studies focused on the exposure of irinotecan in special populations. Yoshida et al. examined mechanisms of PK alterations in patients using irinotecan while Fujita et al. conducted a more recent study that assessed irinotecan's mechanism for delayed SN-38 elimination [34, 35]. In addition, Thai et al. used PBPK modeling to predict PK profiles of docetaxel in children by modulating age-dependent physiological differences [36].

Although the majority of studies cited here utilized a PBPK model, two specific studies were directed towards PKPD modeling. Another study by Chalret du Rieu et al. used a similar methodology to determine maximum tolerated doses of abexinostat in lymphoma and solid tumor patients [2].

\section{Application for Cardiovascular Medications}

Because cardiovascular disease is prevalent throughout much of the developed world, cardiovascular drugs continue to be studied in a variety of patient populations with comorbid disease states to improve outcomes and reduce mortality. Our findings included modeling studies performed on several commonly used drug classes, including HMG-CoA reductase inhibitors, phosphodiesterase- 3 inhibitors, class III antiarrhythmics, angiotensin-receptor blockers, alpha antagonists, beta blockers, and anticoagulants. HMG-CoA reductase inhibitors (also known as statins) are used to reduce the risk of atherosclerotic cardiovascular disease by modulating levels of cholesterol in the blood. This class of medications is notable for its potential of serious adverse effects, including hepatotoxicity, teratogenicity, and myopathy. Statins were the subject of a study by Tsamandouras et al., which predicted the concentration-time profiles of simvastatin in muscle (to assess toxicity) and in the liver (to assess efficacy) [37]. Another class of medications utilized in the literature is the angiotensin-receptor blocking agents (ARBs), which are commonly used for the management of hypertension and heart failure. Li et al. developed a PBPK model which can be used to accurately predict the kinetics of IV telmisartan that incorporated data from sandwich human hepatocyte and liver microsome assays [38].

As the rates of hypertension and cardiovascular disease continue to increase, it is important to continue to construct PBPK models examining the potential drug-drug interactions of these medications. Cardiovascular drugs are often used in combination with each other in a variety of metabolic disease states, and many patients with cardiovascular disease have comorbidities such as diabetes and renal disease, which can impact drug disposition and increase the likelihood of a significant drug interaction. Several studies used modeling to assess the impact of enzyme and transporter-mediated DDIs. Burt et al. investigated the DDI potential of organic cation transporter (OCT) and multi-antimicrobial extrusion protein (MATE) inhibitors between metformin and cimetidine [39]. Fan et al. predicted the mean active metabolite pharmacokinetic profiles in patients taking prasugrel in the presence and absence of a proton pump inhibitor [40]. Ohtani et al. evaluated the concomitant use of bucolome (a CYP2C9 inhibitor) with warfarin [41]. Other studies examined the DDI potential in special populations. For example, Ismail created a DDI model of rivaroxaban and verapamil to investigate the changes in kinetics between healthy subjects and those with renal impairment [42].

Drug-disease modeling is another recognizable advantage to PBPK modeling as drug- and patient-specific parameters may be investigated to better understand altered pharmacokinetics for agents in distinct disease states. Rasool developed a PBPK model that incorporated hepatic and renal hemodynamic changes in patients using carvedilol for chronic heart failure [43]. Vogt developed a PBPK drug-disease model for milrinone in pediatric patients with and without low cardiac output syndrome (LCOS) after open heart surgery in order to provide guidance on optimal dosing [44]. Age dependency of milrinone plasma clearance has been described utilizing nonlinear mixed effects modeling (non-MEM), which is not reflected by age-stratified dosing, and further does not consider differences in organ function between patients with and without LCOS. The study incorporated a drug model consisting of drug-dependent pharmacokinetic and pharmacodynamic properties and a system component for whole-body (i.e., multiple organ compartments) application of intravenous dosing. A disease model with pre-, intra-, and post-operative components associated with open heart surgery consisting of influential factors (i.e., LCOS, acute kidney injury, surgical trauma) was used to describe altered pharmacokinetics of milrinone with and without LCOS. These two models were integrated for simulation in a patient population with varied characteristics, such as age, race, sex, and bodyweight, organ growth, and maturation. The study results generated optimized dosing regimens for LCOS treatment and prevention for pediatric patients stratified into 6 age clusters and provide information that adult dose scaling attempts are inadequate. Furthermore, patients with LCOS vs. those without after surgery require lower maintenance infusion doses. This example of a drug-disease model demonstrates the importance that PBPK modeling can have in balancing harm with efficacy in context of clinical situations requiring higher acuity of care.

Within this same disease state, we can also consider a separate, but clear value from PBPK modeling, which is the employment of quantitative in vitro to in vivo extrapolation (IVIVE). A mainstay within PBPK modeling is the ability to include in vitro experimental observations in order to predict drug disposition within in vivo biological systems, especially 
when such in vivo is not easily available [45]. Marsousi et al. demonstrated increased understanding of ticagrelor pharmacokinetics utilizing PBPK modeling to assess a clinically relevant drug-drug interaction and need for reduced dosing with ritonavir, an antiretroviral agent commonly utilized in HIV and major CYP3A4 inhibitor [46]. Part of this group's assessment was construction of a PBPK model for ticagrelor using intrinsic clearances obtained from in vitro assessments using human liver microsomes [47]. When applying these in vitro values to their model, oral clearance and the exact contribution of CYP3A4 to hepatic metabolism were not wholly accounted for. As such, these model features were refined and improved based on existing clinical literature using sensitivity analyses. The construction of the ticagrelor model coupled with DDI simulation in this example demonstrates how IVIVE can be used to prospectively assess drug disposition for clinically relevant situations to inform dosing decisions.

\section{Application for Neurologic Medications}

DDIs are of great importance with neurologic agents, especially the antiepileptic drugs (AEDs). The AEDs are associated with more DDIs than any other therapeutic drug class, which creates a major therapeutic challenge [48-50]. Currently, about $60-70 \%$ of newly diagnosed patients have their seizures controlled effectively with AED monotherapy; however, the remaining $30-40 \%$ of the patients present with refractory seizures and inevitably need to be treated with AED polytherapy $[51,52]$. Polytherapy with two or more AEDs and/or drugs used for other disease states dramatically increases the probability of DDIs and the risk of serious clinical consequences. Our group has developed PBPK models addressing DDIs of the commonly used AEDs, valproic acid, and lamotrigine $[53,54]$. These models utilize UGT enzyme kinetics from in vitro literature, which have been scaled to fit the observed clinical data. Commonly encountered DDIs of these agents are evaluated such as UGT induction by concomitant administration of other AEDs (e.g., carbamazepine, phenobarbital), as well as the classic UGT inhibition of lamotrigine metabolism by valproic acid.

Prediction of the rate and extent of oral absorption is considered a complex process, which is not easily demonstrated by static models. PBPK modeling is adept to handling prediction of absorption through use of transit models. These models describe the release, dissolution, degradation, metabolism, uptake, and absorption of a molecular entity as it travels through different segments of the GI tract. Simulation incorporates input parameters such as drug solubility, permeability, $\mathrm{pKa}$, LogP, and particle size, as well as diffusion layer models which assimilate differential equations to predict absorption kinetics, guided by Fick's laws of diffusion, across the gut wall. Different commercial simulation packages are available for prediction of absorption, such as GastroPlus ${ }^{\mathrm{TM}}$ advanced compartmental absorption transit (ACAT) model, PK-SIM $®$ absorption model, and SimCYPC's advanced dissolution, absorption, and metabolism (ADAM) model [55, 56]. Our group has utilized SimCYPC's ADAM model to demonstrate the absorption kinetic profile of lamotrigine and valproic acid oral extended-release formulations, which allow for more convenient once-daily dosing of crucial anticonvulsant medication to patients with epilepsy $[53,54]$. Furthermore, these formulations reduce serum concentration peak-to-trough by 10 $20 \%$ therefore minimizing unwanted peak adverse effects and maximizing concentration-dependent efficacy profiles.

DDI studies are of particular interest for the opioid receptor modulating agents, as increased exposure to these agents has the potential to lead to serious adverse effects including respiratory depression and death. Marsousi et al. have developed a model of inhibition of oxycodone metabolism, and its two primary metabolites, in the context of CYP2D6 extensive and poor metabolizers, as well as CYP2D6 and CYP3A4 inhibitors [57]. A second study by Zhou et al. examined the interaction potential of naloxegol, a peripherally acting muopioid receptor antagonist used for the treatment of opioidinduced constipation and a substrate of CYP3A4/3A5 and the P-glycoprotein efflux pump [58]. The results of this study predicted an approximately 13-fold increase in naloxegol exposure when co-administered with ketoconazole, a 3-fold increase in exposure when administered with diltiazem, and a $75 \%$ reduction in naloxegol concentration when administered with rifampin. These models demonstrate the importance of assessing the impact of coadministration of multiple inhibitors together on substrate drugs.

There were several notable examples of neurologic simulations involving special populations. Dosing regimens in pregnant women are currently based on those designed for non-pregnant women, which may not take into account drug distribution and therefore exposure to the fetus. Kalluri et al. developed a PBPK model to assess the kinetics of buprenorphine, a partial agonist at the mu-opioid receptor and a staple in medication-assisted treatment for opioid use disorder, following intravenous and sublingual administration. The results from this study were utilized in a separate study by Zhang et al. to develop a dosing strategy in pregnancy [59, 60]. The model predicted decreased drug exposure and suggest a need for increased dose or frequency in this population, which was consistent with the available literature. This work demonstrates the utility of PBPK modeling for neurologic agents in special populations and the ability to optimize pharmacotherapy without requiring intensive sampling. A second example of PBPK modeling for special populations utilizing neurologic agents is represented in a model for atomoxetine [61]. This work by Huang et al. was developed to determine if a PBPK model for atomoxetine in healthy individuals could be extrapolated to special populations (e.g., renal or hepatic 
impairment, ethnicity, pediatrics). The model demonstrated predictive performance in Asian and pediatric patients; however, AUC was over-predicted 3- to 4-fold in both end-stage renal disease and hepatic impairment subjects. The results indicate that modeling in healthy individuals can be extrapolated to other certain special populations; however, predictive performance requires optimization in key end organ disease states.

\section{Application for Miscellaneous}

Therapeutic areas representing altogether $\leq 14 \%$ were renal, hepatic, respiratory, gastrointestinal, immunosuppressants, supplements, and miscellaneous. A major focus of these studies was DDI and transporter-mediated effects of drug concentration. For DDI prediction, Duan et al. assessed CYP2C19 ontogeny in neonates and infants [62]. Several endocrine topics were examined including repaglinide's inhibitory effect on the metabolism of pioglitazone [63]. Other studies examined the impact of DDIs on several medications which are major substrates of cytochrome P450 enzymes in children [58]. In addition, several studies analyzed hepatic and renal elimination of medications. The role of organic anion transporter-2 in the hepatic uptake of tolbutamide was studied by Bi et al. [64]. A 2018 study by Nakada et al. determined if the elevation of serum creatinine could be explained by inhibition by trimethoprim on tubular secretion of creatinine via renal transporters such as OCT2, OCT3, MATE1, and MATE2-K, while Tan et al. examined the impact of [65] kidney disease on the disposition of hepatic CYP2C8 and OATP1B drug substrates [66].

\section{Discussion}

Medication safety is, and continues to be, a major concern throughout all aspects of healthcare. All drugs have adverse effects, which can range from acute and mild (e.g., itching, headache) to severe and chronic (e.g., hepatotoxicity, druginduced lupus erythematosus). In order to minimize adverse effects and optimize clinical outcomes, healthcare providers must consider a drug's indication and clinical efficacy but must also identify potentially hazardous drug-drug interactions and engage in monitoring for drug-related toxicities. However, drug dosing is not straightforward, and patientspecific factors such as weight, age, renal/hepatic function, and genetic polymorphisms can lead to unanticipated drug effects, especially with drugs that have a narrow therapeutic window. Because many large clinical trials exclude patients with varying degrees of renal/hepatic dysfunction, extremes of age, and other distinguishing characteristics as a means to promote safety, providers who are involved in the care of special populations are often left to case reports and animal studies to assess the risks and benefits of therapy in their patients. Our review examined how simulated modeling of drug pharmacokinetics is also being utilized for this purpose, and our results show an increase in the utilization of PBPK modeling within the last decade, particularly for predicting the outcomes of drug-drug interactions and understanding how kinetics are altered in special populations of patients who are often times underrepresented in large-scale clinical trials.

Our results demonstrate that the majority of developed PBPK models consist of those for infectious disease purposes (24\%), followed by those for oncology (22\%) and neurology $(18 \%)$. In addition, the therapeutic areas which experienced the highest proportions of growth during this time period were infectious diseases and neurology, experiencing a 16-fold and 12-fold increase in the annual number of publications, respectively. The increase in models within these therapeutic areas over the last several years is suggestive of efforts to maintain better safety and efficacy profiles for drugs with narrow therapeutic indices and/or difficult-to-predict doses. Our results show that the utilization of modeling for a particular disease state appears to be inversely proportional to the prevalence of that disease state, meaning that the number of published articles increases as the number of patients available to participate in clinical studies decreases. The abundance of oncologically focused studies is due in part to the challenges with feasibility of clinical studies involving these medications (e.g., low patient population, chronic disease progress which makes follow-up difficult), but also because patients with cancer have a variety of comorbidities which can influence drug disposition, such as renal/hepatic impairment, immune deficiency, GI absorption issues, etc. For patients with cancer, PBPK modeling offers greater control over patient parameters compared to clinical trials and can be used to guide dosing strategies in patients with a multitude of comorbidities.

Our literature review highlighted several high-impact applications of PBPK modeling in the drug development process which have led to revisions in a drug's prescribing information and package insert. Drug-drug interaction and bioavailability studies were included in the package insert information for ribociclib, a cyclin-dependent kinase 4/6 inhibitor approved by the FDA in 2017 for the treatment of breast cancer. In this instance, the authors utilized Simcyp for the prediction of ribociclib exposure in the presence of CYP3A4 inhibitors. Based on their findings, they made a formal recommendation to amend the package insert to state that ribociclib should be avoided in the presence of CYP3A4 inhibitors/inducers. Similarly, literature contributed by Arya and Shebley was used to recommend an update to the package labeling for dasabuvir, a non-nucleoside inhibitor of the hepatitis $C$ virus's RNA-dependent RNA polymerase, due to their modeling efforts which showed a dangerous increase in drug exposure in the presence of CYP2C8 inhibitors [67, 68]. 
The increase in PBPK modeling could also be due to guidance from the FDA suggesting that PBPK modeling is suited for DDI assessment and hepatic impairment studies. A statement by the Center for Drug Evaluation and Research of the FDA stated that "the decision to accept results from PBPK analyses in lieu of clinical pharmacokinetic data is made on a case-by-case basis, considering the intended uses as well as the quality, relevance, and reliability of results from the PBPK analyses". The guidance letter goes on to state that PBPK studies are permitted to be utilized in lieu of prospective DDI studies for investigational drugs and moderate-to-weak inhibitors of their metabolism provided that these models can be verified using human PK data and prospective DDI studies which investigated the effects of strong inhibitors or inducers.

There are several factors which have limited the adoption of PBPK modeling in various therapeutic areas, such as cost and ease-of-use. A commercial license for simulation programs can cost upwards of $\$ 100,000$ per year, and the time required to familiarize researchers with the user interface of these programs is a financial and labor-intensive strain which many organizations may not be willing to invest in. Another major barrier to use has been the challenges associated with model validation. Because PBPK modeling is geared generally towards utilization in difficult-to-predict clinical scenarios or situations where obtaining human data is unethical or impractical, there is a great deal of disagreement on the appropriate strategies for model validation. Currently, model validation is accomplished with data from animal studies, which is not always a reliable predictor of kinetics in humans or based on human data from pharmacokinetic studies on drugs which are similar to the study drug.

This review article is the first to highlight PBPK articles over the past several years on major trends in therapeutic area, applications, and drug class. In addition to highlighting some of the high-impact applications of PBPK modeling, we also examined the various regulatory and logistical barriers which have limited the utilization of this practice in certain therapeutic areas. We did not utilize a standardized assessment tool for each of the articles; rather, the information that we needed for the review was pulled from the abstract and, where necessary, the full manuscript. Although we used the most generic search terms possible for our literature search, it is unlikely that we were able to pull and analyze all of the qualifying articles published from 2012 to 2018. In addition, by limiting the scope of our articles to products which are currently approved by the FDA, it is likely that we may have bypassed articles on drugs which are currently in development, although it is unclear the extent.

\section{Conclusion}

This study has shown a dramatic increase in the number of PBPK papers written from 2012 to 2018, with an emphasis on oncological drugs and applications regarding evaluation of DDIs. While several review papers published recently have focused on assessing the predictability of PBPK modeling with regard to drug-drug interactions, absorption, food effects, etc., this review primarily summarizes the application of PBPK modeling for FDA-approved drugs across various therapeutic areas. This study is beneficial to assess the increasing use of PBPK modeling in therapeutic drug monitoring, especially in relation to narrow therapeutic index drugs within oncologic, neurologic, antimicrobial, and cardiovascular drug classes. Future research includes further insight into the magnitude of studies influencing package insert dosing recommendations and the distribution of these between PK prediction, DDI, and special population applications. Furthermore, assessment of simulation interfaces to support clinical utility of PBPK modeling is needed in order to streamline this process for practice in a healthcare setting.

Data Availability The other data of the current paper is available upon request.

\section{Compliance with Ethical Standards}

Conflict of Interest On behalf of all authors, the corresponding author states that there is no conflict of interest.

Human and Animal Rights and Informed Consent This article does not contain any studies with human or animal subjects performed by any of the authors.

\section{References}

1. Murdaugh L. Adverse drug reaction reporting. Competence Tools for Health-Systems Pharmacists. p. 545-56.

2. Chalret du Rieu Q, Fouliard S, White-Koning M, Kloos I, Chatelut E, Chenel M. Pharmacokinetic/pharmacodynamic modeling of abexinostat-induced thrombocytopenia across different patient populations: application for the determination of the maximum tolerated doses in both lymphoma and solid tumour patients. Investig New Drugs. 2014;32(5):985-94. https://doi.org/10.1007/s10637-0140118-1.

3. Mayo PR, Ling SY, Huizinga RB, Freitag DG, Aspeslet LJ, Foster RT. Population PKPD of voclosporin in renal allograft patients. J Clin Pharmacol. 2014;54(5):537-45. https://doi.org/10.1002/jcph. 237.

4. Narayanan R, Hoffmann M, Kumar G, Surapaneni S. Application of a "fit for purpose" PBPK model to investigate the CYP3A4 induction potential of enzalutamide. Drug Metab Lett. 2016;10(3):172-9. https://doi.org/10.2174/ 1872312810666160729124745.

5. Sager JE, Yu J, Ragueneau-Majlessi I, Isoherranen N. Physiologically based pharmacokinetic (PBPK) modeling and simulation approaches: a systematic review of published models, applications, and model verification. Drug Metab Dispos. 2015;43(11):1823-37. https://doi.org/10.1124/dmd.115.065920.

6. Walsh C, Bonner JJ, Johnson TN, Neuhoff S, Ghazaly EA, Gribben $\mathrm{JG}$, et al. Development of a physiologically based pharmacokinetic 
model of actinomycin D in children with cancer. Br J Clin Pharmacol. 2016;81(5):989-98. https://doi.org/10.1111/bcp.12878.

7. Diestelhorst C, Boos J, McCune JS, Russell J, Kangarloo SB, Hempel G. Predictive performance of a physiologically based pharmacokinetic model of busulfan in children. Pediatr Hematol Oncol. 2014;31(8):731-42. https://doi.org/10.3109/08880018.2014. 927945.

8. FDA. Physiologically based pharmacokinetic analyses - format and content guidance for industry. US Food and Drug Administration. 2019.

9. EMA. Reporting of physiologically based pharmacokinetic (PBPK) modelling and simulation. European Medicines Agency. 2018.

10. Jamei M. Recent advances in development and application of physiologically-based pharmacokinetic (PBPK) models: a transition from academic curiosity to regulatory acceptance. Curr Pharmacol Rep. 2016;2(3):161-9. https://doi.org/10.1007/s40495016-0059-9.

11. Teorell T, STUDIES ON. The diffusion effect upon ionic distribution : ii. Experiments on ionic accumulation. J Gen Physiol. 1937;21(1):107-22.

12. Teorell T. Kinetics of distribution of substances administered to the body, I: the extravascular modes of administration. Archives Internationales de Pharmacodynamie et de Therapie. 1937;57: 205-25.

13. Roberts $\mathrm{O}$, Khoo $\mathrm{S}$, Owen $\mathrm{A}$, Siccardi M. Interaction of rifampin and darunavir-ritonavir or darunavir-cobicistat in vitro. Antimicrob Agents Chemother. 2017;61(5). doi:https://doi.org/10.1128/AAC. 01776-16.

14. Kersting G, Willmann S, Wurthwein G, Lippert J, Boos J, Hempel G. Physiologically based pharmacokinetic modelling of high- and low-dose etoposide: from adults to children. Cancer Chemother Pharmacol. 2012;69(2):397-405. https://doi.org/10.1007/s00280011-1706-9.

15. Rizk ML, Zou L, Savic RM, Dooley KE. Importance of drug pharmacokinetics at the site of action. Clin Transl Sci. 2017;10(3):13342. https://doi.org/10.1111/cts. 12448 .

16. de Lange EC, Hammarlund-Udenaes M. Translational aspects of blood-brain barrier transport and central nervous system effects of drugs: from discovery to patients. Clin Pharmacol Ther. 2015;97(4):380-94. https://doi.org/10.1002/cpt.76.

17. Henderson JT, Piquette-Miller M. Blood-brain barrier: an impediment to neuropharmaceuticals. Clin Pharmacol Ther. 2015;97(4): 308-13. https://doi.org/10.1002/cpt.77.

18. Almond LM, Yang J, Jamei M, Tucker GT, Rostami-Hodjegan A. Towards a quantitative framework for the prediction of DDIs arising from cytochrome P450 induction. Curr Drug Metab. $2009 ; 10(4): 420-32$. https://doi.org/10.2174/ 138920009788498978.

19. Rostami-Hodjegan A, Tucker GT. Simulation and prediction of in vivo drug metabolism in human populations from in vitro data. Nat Rev Drug Discov. 2007;6(2):140-8. https://doi.org/10.1038/ $\operatorname{nrd} 2173$.

20. Rowland M, Peck C, Tucker G. Physiologically-based pharmacokinetics in drug development and regulatory science. Annu Rev Pharmacol Toxicol. 2011;51:45-73. https://doi.org/10.1146/ annurev-pharmtox-010510-100540.

21. Schlender JF, Teutonico D, Coboeken K, Schnizler K, Eissing T, Willmann S, et al. A physiologically-based pharmacokinetic model to describe ciprofloxacin pharmacokinetics over the entire span of life. Clin Pharmacokinet. 2018;57(12):1613-34. https://doi.org/10. 1007/s40262-018-0661-6.

22. Claassen K, Thelen K, Coboeken K, Gaub T, Lippert J, Allegaert K, et al. Development of a physiologically-based pharmacokinetic model for preterm neonates: evaluation with in vivo data. Curr
Pharm Des. 2015;21(39):5688-98. https://doi.org/10.2174/ 1381612821666150901110533.

23. Hornik CP, Wu H, Edginton AN, Watt K, Cohen-Wolkowiez M, Gonzalez D. Development of a pediatric physiologically-based pharmacokinetic model of clindamycin using opportunistic pharmacokinetic data. Clin Pharmacokinet. 2017;56(11):1343-53. https://doi.org/10.1007/s40262-017-0525-5.

24. Marzolini C, Rajoli R, Battegay M, Elzi L, Back D, Siccardi M. Physiologically based pharmacokinetic modeling to predict drugdrug interactions with efavirenz involving simultaneous inducing and inhibitory effects on cytochromes. Clin Pharmacokinet. 2017;56(4):409-20. https://doi.org/10.1007/s40262-016-0447-7.

25. Roberts O, Rajoli RKR, Back DJ, Owen A, Darin KM, Fletcher CV, et al. Physiologically based pharmacokinetic modelling prediction of the effects of dose adjustment in drug-drug interactions between levonorgestrel contraceptive implants and efavirenz-based ART. J Antimicrob Chemother. 2018;73(4):1004-12. https://doi.org/10. 1093/jac/dkx515.

26. Yao X, Ye F, Zhang M, Cui C, Huang B, Niu P, et al. In vitro antiviral activity and projection of optimized dosing design of hydroxychloroquine for the treatment of severe acute respiratory syndrome coronavirus 2 (SARS-CoV-2). Clin Infect Dis. 2020. https://doi.org/10.1093/cid/ciaa237.

27. Thompson MD, Beard DA. Physiologically based pharmacokinetic tissue compartment model selection in drug development and risk assessment. J Pharm Sci. 2012;101(1):424-35. https://doi.org/10. 1002/jps. 22768

28. Einolf HJ, Zhou J, Won C, Wang L, Rebello S. A physiologicallybased pharmacokinetic modeling approach to predict drug-drug interactions of sonidegib (LDE225) with perpetrators of CYP3A in cancer patients. Drug Metab Dispos. 2017;45(4):361-74. https://doi.org/10.1124/dmd.116.073585.

29. Budha NR, Ji T, Musib L, Eppler S, Dresser M, Chen Y, et al. Evaluation of cytochrome P450 3A4-mediated drug-drug interaction potential for cobimetinib using physiologically based pharmacokinetic modeling and simulation. Clin Pharmacokinet. 2016;55(11):1435-45. https://doi.org/10.1007/s40262-016-04125.

30. Yamazaki S, Johnson TR, Smith BJ. Prediction of drug-drug interactions with crizotinib as the CYP3A substrate using a physiologically based pharmacokinetic model. Drug Metab Dispos. 2015;43(10):1417-29. https://doi.org/10.1124/dmd.115.064618.

31. Posada MM, Bacon JA, Schneck KB, Tirona RG, Kim RB, Higgins $\mathrm{JW}$, et al. Prediction of renal transporter mediated drug-drug interactions for pemetrexed using physiologically based pharmacokinetic modeling. Drug Metab Dispos. 2015;43(3):325-34. https://doi. org/10.1124/dmd.114.059618.

32. Toshimoto K, Tomaru A, Hosokawa M, Sugiyama Y. Virtual clinical studies to examine the probability distribution of the AUC at target tissues using physiologically-based pharmacokinetic modeling: application to analyses of the effect of genetic polymorphism of enzymes and transporters on irinotecan induced side effects. Pharm Res. 2017;34(8):1584-600. https://doi.org/10.1007/s11095-0172153-z.

33. Dickschen KJ, Willmann S, Hempel G, Block M. Addressing adherence using genotype-specific PBPK modeling-impact of drug holidays on tamoxifen and endoxifen plasma levels. Front Pharmacol. 2017;8:67. https://doi.org/10.3389/fphar.2017.00067.

34. Fujita K, Masuo Y, Okumura H, Watanabe Y, Suzuki H, Sunakawa $\mathrm{Y}$, et al. Increased plasma concentrations of unbound SN-38, the active metabolite of irinotecan, in cancer patients with severe renal failure. Pharm Res. 2016;33(2):269-82. https://doi.org/10.1007/ s11095-015-1785-0.

35. Yoshida K, Maeda K, Kusuhara H, Konagaya A. Estimation of feasible solution space using cluster Newton method: application to pharmacokinetic analysis of irinotecan with physiologically- 
based pharmacokinetic models. BMC Syst Biol. 2013;7 Suppl 3: S3. https://doi.org/10.1186/1752-0509-7-S3-S3.

36. Thai HT, Mazuir F, Cartot-Cotton S, Veyrat-Follet C. Optimizing pharmacokinetic bridging studies in paediatric oncology using physiologically-based pharmacokinetic modelling: application to docetaxel. Br J Clin Pharmacol. 2015;80(3):534-47. https://doi. org/10.1111/bcp.12702.

37. Tsamandouras N, Dickinson G, Guo Y, Hall S, Rostami-Hodjegan A, Galetin A, et al. Identification of the effect of multiple polymorphisms on the pharmacokinetics of simvastatin and simvastatin acid using a population-modeling approach. Clin Pharmacol Ther. 2014;96(1):90-100. https://doi.org/10.1038/clpt.2014.55.

38. Li R, Ghosh A, Maurer TS, Kimoto E, Barton HA. Physiologically based pharmacokinetic prediction of telmisartan in human. Drug Metab Dispos. 2014;42(10):1646-55. https://doi.org/10.1124/ dmd.114.058461.

39. Burt HJ, Neuhoff S, Almond L, Gaohua L, Harwood MD, Jamei M, et al. Metformin and cimetidine: physiologically based pharmacokinetic modelling to investigate transporter mediated drug-drug interactions. Eur J Pharm Sci. 2016;88:70-82. https://doi.org/10. 1016/j.ejps.2016.03.020.

40. Fan J, Zhang X, Zhao L. Utility of physiologically based pharmacokinetic absorption modeling to predict the impact of aalt-To-base conversion on prasugrel $\mathrm{HCl}$ product bioequivalence in the presence of proton pump inhibitors. AAPS J. 2017;19(5):1479-86. https://doi.org/10.1208/s12248-017-0116-2.

41. Ohtani H, Hakoda R, Imaoka A, Akiyoshi T. In silico evaluation of warfarin-bucolome therapy. Biopharm Drug Dispos. 2016;37(4): 233-42. https://doi.org/10.1002/bdd.2008.

42. Ismail M, Lee VH, Chow CR, Rubino CM. Minimal physiologically based pharmacokinetic and drug-drug-disease interaction model of rivaroxaban and verapamil in healthy and renally impaired subjects. J Clin Pharmacol. 2018;58(4):541-8. https://doi.org/10. 1002/jcph.1044.

43. Rasool MF, Khalil F, Laer S. Optimizing the clinical use of carvedilol in liver cirrhosis using a physiologically based pharmacokinetic modeling approach. Eur J Drug Metab Pharmacokinet. 2017;42(3):383-96. https://doi.org/10.1007/s13318-016-0353-2.

44. Vogt W. Evaluation and optimisation of current milrinone prescribing for the treatment and prevention of low cardiac output syndrome in paediatric patients after open heart surgery using a physiology-based pharmacokinetic drug-disease model. Clin Pharmacokinet. 2014;53(1):51-72. https://doi.org/10.1007/ s40262-013-0096-z.

45. Yoon M, Campbell JL, Andersen ME, Clewell HJ. Quantitative in vitro to in vivo extrapolation of cell-based toxicity assay results. Crit Rev Toxicol. 2012;42(8):633-52. https://doi.org/10.3109/ 10408444.2012.692115.

46. Marsousi N, Samer CF, Fontana P, Reny JL, Rudaz S, Desmeules JA, et al. Coadministration of ticagrelor and ritonavir: toward prospective dose adjustment to maintain an optimal platelet inhibition using the PBPK approach. Clin Pharmacol Ther. 2016;100(3):295304. https://doi.org/10.1002/cpt.407.

47. Zhou D, Andersson TB, Grimm SW. In vitro evaluation of potential drug-drug interactions with ticagrelor: cytochrome P450 reaction phenotyping, inhibition, induction, and differential kinetics. Drug Metab Dispos. 2011;39(4):703-10. https://doi.org/10.1124/dmd. 110.037143

48. Patsalos PN. Drug interactions with the newer antiepileptic drugs (AEDs)-part 1: pharmacokinetic and pharmacodynamic interactions between AEDs. Clin Pharmacokinet. 2013;52(11):927-66. https://doi.org/10.1007/s40262-013-0087-0.

49. Patsalos PN. Drug interactions with the newer antiepileptic drugs (AEDs)-part 2: pharmacokinetic and pharmacodynamic interactions between AEDs and drugs used to treat non-epilepsy disorders.
Clin Pharmacokinet. 2013;52(12):1045-61. https://doi.org/10. 1007/s40262-013-0088-z.

50. Patsalos PN, Froscher W, Pisani F, van Rijn CM. The importance of drug interactions in epilepsy therapy. Epilepsia. 2002;43(4):36585. https://doi.org/10.1046/j.1528-1157.2002.13001.x.

51. Brodie MJ, Mintzer S, Pack AM, Gidal BE, Vecht CJ, Schmidt D. Enzyme induction with antiepileptic drugs: cause for concern? Epilepsia. 2013;54(1):11-27. https://doi.org/10.1111/j.1528-1167. 2012.03671.x.

52. Kwan P, Brodie MJ. Epilepsy after the first drug fails: substitution or add-on? Seizure. 2000;9(7):464-8. https://doi.org/10.1053/seiz. 2000.0442.

53. Conner TM, Nikolian VC, Georgoff PE, Pai MP, Alam HB, Sun D, et al. Physiologically based pharmacokinetic modeling of disposition and drug-drug interactions for valproic acid and divalproex. Eur J Pharm Sci. 2018;111:465-81. https://doi.org/10.1016/j.ejps. 2017.10.009.

54. Conner TM, Reed RC, Zhang T. A physiologically based pharmacokinetic model for optimally profiling lamotrigine disposition and drug-drug interactions. Eur J Drug Metab Pharmacokinet. 2019;44(3):389-408. https://doi.org/10.1007/s13318-018-0532-4.

55. Jones HM, Gardner IB, Watson KJ. Modelling and PBPK simulation in drug discovery. AAPS J. 2009;11(1):155-66. https://doi.org/ 10.1208/s12248-009-9088-1.

56. Jamei M, Turner D, Yang J, Neuhoff S, Polak S, Rostami-Hodjegan A, et al. Population-based mechanistic prediction of oral drug absorption. AAPS J. 2009;11(2):225-37. https://doi.org/10.1208/ s12248-009-9099-y.

57. Huang X-F, Tang J-F, Ji J-L, Wang X-L, Ruan B-F. Synthesis, characterization and antitumor activity of novel amide derivatives containing ferrocenyl pyrazol-moiety. Journal of Organometallic Chemistry. 2012;706-707:113-23.

58. Zhou W, Johnson TN, Bui KH, Cheung SYA, Li J, Xu H, et al. Predictive performance of physiologically based pharmacokinetic (PBPK) modeling of drugs extensively metabolized by major cytochrome P450s in children. Clin Pharmacol Ther. 2018;104(1):188200. https://doi.org/10.1002/cpt.905.

59. Kalluri HV, Zhang H, Caritis SN, Venkataramanan R. A physiologically based pharmacokinetic modelling approach to predict buprenorphine pharmacokinetics following intravenous and sublingual administration. Br J Clin Pharmacol. 2017;83(11):2458-73. https://doi.org/10.1111/bcp.13368.

60. Zhang H, Kalluri HV, Bastian JR, Chen H, Alshabi A, Caritis SN, et al. Gestational changes in buprenorphine exposure: a physiologically-based pharmacokinetic analysis. Br J Clin Pharmacol. 2018;84(9):2075-87. https://doi.org/10.1111/bcp. 13642.

61. Huang W, Nakano M, Sager J, Ragueneau-Majlessi I, Isoherranen N. Physiologically based pharmacokinetic model of the CYP2D6 probe atomoxetine: extrapolation to special populations and drugdrug interactions. Drug Metab Dispos. 2017;45(11):1156-65. https://doi.org/10.1124/dmd.117.076455.

62. Duan P, Wu F, Moore JN, Fisher J, Crentsil V, Gonzalez D, et al. Assessing CYP2C19 ontogeny in neonates and infants using physiologically based pharmacokinetic models: impact of enzyme maturation versus inhibition. CPT Pharmacometrics Syst Pharmacol. 2019;8(3):158-66. https://doi.org/10.1002/psp4.12350.

63. Xiao Q, Tang L, Xu R, Qian W, Yang J. Physiologically based pharmacokinetics model predicts the lack of inhibition by repaglinide on the metabolism of pioglitazone. Biopharm Drug Dispos. 2015;36(9):603-12. https://doi.org/10.1002/bdd.1987.

64. Bi YA, Mathialagan S, Tylaska L, Fu M, Keefer J, Vildhede A, et al. Organic anion transporter 2 mediates hepatic uptake of tolbutamide, a CYP2C9 probe drug. J Pharmacol Exp Ther. 2018;364(3):390-8. https://doi.org/10.1124/jpet.117.245951. 
65. Nakada T, Kudo T, Kume T, Kusuhara H, Ito K. Quantitative analysis of elevation of serum creatinine via renal transporter inhibition by trimethoprim in healthy subjects using physiologically-based pharmacokinetic model. Drug Metab Pharmacokinet. 2018;33(1): 103-10. https://doi.org/10.1016/j.dmpk.2017.11.314.

66. Tan ML, Zhao P, Zhang L, Ho YF, Varma MVS, Neuhoff S, et al. Use of physiologically based pharmacokinetic modeling to evaluate the effect of chronic kidney disease on the disposition of hepatic CYP2C8 and OATP1B drug substrates. Clin Pharmacol Ther. 2019;105(3):719-29. https://doi.org/10.1002/cpt.1205.

67. Arya V, Zhao P, Reynolds KS, Mishra P, Younis IR. Utilizing PBPK modeling to evaluate the potential of a significant drug-drug interaction between clopidogrel and dasabuvir: a scientific perspective. Clin Pharmacol Ther. 2017;102(4):578-80. https://doi.org/10. 1002/cpt.699.

68. Shebley M, Fu W, Badri P, Bow D, Fischer V. Physiologically based pharmacokinetic modeling suggests limited drug-drug interaction between clopidogrel and dasabuvir. Clin Pharmacol Ther. 2017;102(4):679-87. https://doi.org/10.1002/cpt.689.

Publisher's Note Springer Nature remains neutral with regard to jurisdictional claims in published maps and institutional affiliations. 